\title{
INVARIANT METRICS ON TEICHMÜLLER SPACES AND QUASICONFORMAL EXTENDABILITY OF ANALYTIC FUNCTIONS
}

\author{
S. L. KRUŠKAL,
}

1. Introductory remarks. Deep connections of the classical geometric function theory with the general methods of multidimensional complex analysis and holomorphic fiberings above Teichmüller spaces have been discovered lately. Some related results are given in the present paper.

First of all, the problem concerning the relation between Carathéodory and Teichmüller metrics is solved on arbitrary finite-dimensional Teichmüller spaces and the universal Teichmüller space. The key theorems 1 and 2 state that these metrics do not coincide. Hence, as the most important corollary, we obtain the general result that no sufficient condision for the $k$-quasiconformal extendability of analytic functions defined on a simply connected plane domain bounded by a quasicircle (i.e. quasidisc), which is expressed in terms of inequalities for analytic funcionals, can coincide with the necessary ones. As another application, we obtain an essential strengthening of the area principle and its corollaries which play the fundamental role in the univalent function theory.

2. Preliminaries and notations. Denote: $U=\{z=x+i y \in C: \operatorname{Im} z>0\}, U^{*}=$ $\{z \in C: \operatorname{Im} z<0\}, \Delta=\{z \in C:|z|<1\} ; \varrho\left(z^{\prime}, z^{\prime \prime}\right)$ is the hyperbolic metric in $\Delta$ of Gauss curvature $K=-4$, i.e., with the differential element $d \varrho=|d z| /\left(1-|z|^{2}\right)$. Considering functions from $L_{\infty}(U)$ we assume them extended on $U^{*}$ by identical zero. Let $B_{2}\left(U^{*}\right)$ be the complex Banach space of functions holomorphic in $U^{*}$ with the norm $\|\varphi\|=\sup _{U^{*}}|z-\bar{z}|^{2}|\varphi(z)|, A(U)$ be the (closed) subspace in $L_{1}(U)$ formed by integrable holomorphic functions in $U$.

Let $T$ be the universal Teichmüller space which we consider to be realized (up to a biholomorphic isomorphism) as a bounded domain in the space $B_{2}\left(U^{*}\right)$ filled by Schwarzian derivatives

$$
S_{w}=\left(\frac{w^{\prime \prime}(z)}{w^{\prime}(z)}\right)^{\prime}-\frac{1}{2}\left(\frac{w^{\prime \prime}(z)}{w^{\prime}(z)}\right)^{2}
$$

of univalent analytic functions $w(z)$ in $U^{*}$ admitting quasiconformal extension on $U$ (this domain lies in the ball $\{\|\varphi\|<6\}$ ). 
Consider the ball

$$
B(U)=\left\{\mu \in L_{\infty}(C):\left.\mu\right|_{U^{*}}=0, \quad\|\mu\|<1\right\}
$$

and quasiconformal automorphisms $w^{\mu}(z)$ of the Riemann sphere $\overline{\boldsymbol{C}}$ with Beltrami coefficients $\mu \in B(U)$, which leave 0,1 and $\infty$ fixed. Up to an isometric isomorphism $T$ is also the factor of the ball $B(U)$ with respect to the equivalence relation: $\mu \sim v$ if $\left.w^{\mu}\right|_{R}=\left.w^{v}\right|_{R}$, supplied with the structure of the complex Banach manifold by its biholomorphic immersion into $B_{2}\left(U^{*}\right)$ in the above way; then the projection

$$
\Phi(\mu)=S_{W^{\mu}}: B(U) \rightarrow B_{2}\left(U^{*}\right)
$$

is also holomorphic.

On the space $T$ one defines the intrinsic Teichmüller metric $\tau_{T}$, the Carathéodory metric $c_{T}$ and the Kobayashi metric $k_{T}$. Recall that

$$
\tau_{T}(\varphi, \psi)=\frac{1}{2} \inf \log K\left(w^{\mu} \circ\left(w^{v}\right)^{-1}\right)
$$

by $\quad$ all $\quad \mu \in \Phi^{-1}(\varphi), \quad v \in \Phi^{-1}(\psi) \quad\left(K\left(w^{\sigma}\right)=\left(1+\|\sigma\|_{\infty}\right) /\left(1-\|\sigma\|_{\infty}\right)\right), \quad c_{T}(\varphi, \psi)=$ $=\sup \{\varrho(f(\varphi), f(\psi)) \mid f: T \rightarrow \Delta$ holomorphic $\}$ and $k_{T}(\varphi, \psi)$ is the greatest of all pseudometrics $d$ on $T$ satisfying the inequality $d(f(z), f(\zeta)) \leqq \varrho(z, \zeta)$ for all holomorphic $f: \Delta \rightarrow T$.

We shall also consider finite-dimensional Teichmüller spaces $T(g, n)$ for Riemann surfaces of finite type $(g, n), 2 g-2+n>0$, replacing the index $T$ by $T(g, n)$ in the notations of the metrics.

3. Carathéodory and Kobayashi metrics. From the general properties of invariant metrics and extremal quasiconformal mappings it easily follows that for each of the spaces $T$ and $T(g, n)$ the relation

$$
c(\varphi, \psi) \leqq k(\varphi, \psi) \leqq \tau(\varphi, \psi)
$$

holds. Due to Royden's theorem [6], $k_{T(g, n)}=\tau_{T(g, n)}$. To find out when the Carathéodory metric coincides with the majorizing Kobayashi metric is one of the general problems of complex manifolds. For arbitrary Teichmüller spaces such a problem has not been solved yet.

The following two theorems are connected with the solution of this problem and are also the basis for subsequent results.

Theorem 1. In finite-dimensional Teichmüller spaces $T(g, n)$ of dimension exceeding 2 the Carathéodory metric does not coincide with the Teichmüller-Kobayashi hyperbolic metric.

Theorem 2. The Carathéodory metric of the universal Teichmüller space $T$ does not coincide with the Teichmüller metric, namely, in the neighbourhood of every point $\varphi_{0} \in T$ there exist points $\varphi$ for which $c_{T}\left(\varphi, \varphi_{0}\right)<\tau_{T}\left(\varphi, \varphi_{0}\right)$. 
Theorem 1 was proved in the author's paper [1] for closed surfaces with punctures, and in a stronger formulation, similar to Theorem 2 (the detailed presentation is given in [2]). The basic scheme of this proof is also valid for the spaces $T(g, 0)$ of closed surfaces of genus $g \geqq 2$, but it demands some modification of the arguments used in [1], namely, one should pass over to an analytic submanifold in $T(g, 0)$ (of lower dimension for $g>2$ ) formed by hyperelliptic surfaces.

This idea of a "descent" on a submanifold can be used to prove Theorem 2, but, unlike the finite-dimensional case, the situation here gets considerably more complicated. Let us outline the scheme of the proof. The details will be presented elsewhere.

Since the space $T$ is homogeneous, it is sufficient to establish the statement of the theorem for the point $\varphi_{0}=\mathbf{0}$ and to consider only some neighbourhood $N_{0}$ of this point in $T$. Due to the local nature it is more convenient to use the corresponding differential forms $\boldsymbol{c}(\varphi ; \xi), \boldsymbol{k}(\varphi ; \xi), \beta(\varphi ; \xi)$ of the above metrics on tangent bundle $T(T)$ and, moreover, one only needs values of these differential metrics on the tangent space $T_{0}(T)$ of $T$ in $\mathbf{0}$ (note that $\beta$ is a Finsler structure on $T(T)$ generating $\tau_{T}$ ).

Suppose that the equality $c_{T}(\varphi, \mathbf{0})=\tau_{T}(\varphi, \mathbf{0})$ holds for all $\varphi \in N_{0}$. Then we obtain from (2) that for all tangent vectors $\xi \in T_{0}(T)$ there should be

$$
\boldsymbol{c}(\mathbf{0} ; \xi)=\boldsymbol{k}(\mathbf{0} ; \xi)=\beta(\mathbf{0} ; \xi) .
$$

Now fix the points $x_{1}, x_{2}, \ldots, x_{n} \in \boldsymbol{R} \backslash\{0,1\}, n \geqq 3$, and denote by $A_{n}$ a linear hull in $A(U)$ of rational functions

$$
\varphi_{1}(z)=\frac{1}{z(z-1)\left(z-x_{1}\right)}, \ldots, \varphi_{n}(z)=\frac{1}{z(z-1)\left(z-x_{n}\right)} .
$$

For all $\varphi \in A_{n}$ consider in $T$ holomorphic discs $\{\Phi(t \bar{\varphi} /|\varphi|)\},|t|<1$. One can show that these discs fill some $n$-dimensional complex submanifold $V_{n}$ in the neighbourhood of zero in $T$. The proof is based on some results of the theory of quasiconformal mappings and the properties of the differential $d \Phi(\mathbf{0})$ of mapping (1) at zero. Vectors tangent to $V_{n}$ (with respect to the whole $T$ ) determine in $T_{0}(T)$ an $n$-dimensional subspace which we denote by $Q_{n}$. According to equality (3), for every element $\xi \in Q_{n}$ there exists a projector $P_{\xi}: Q_{n} \rightarrow\{t \xi\}, t \in C$, with norm 1 .

The basic goal now is to prove that in fact a projector for all $\xi$ cannot exist; hence there follows the contradiction with the accepted assumption $c_{T}=\tau_{T}$. To do this, we construct the operator $P_{\xi}^{*}$ conjugated with $P_{\xi}$ and acting on the corresponding $n$-dimensional sutspace of the cotangent space $T_{0}^{*}(T)$ which is isometrically isomorphic to $A_{n}$. Further arguments are presented by the scheme similar to that used in [1]. One can verify that for all $\varphi=a_{1} \varphi_{1}+a_{2} \psi$, where $\left(a_{1}, a_{2}\right) \in \boldsymbol{C}^{2}$,

$$
\psi(z)=\left(z-x_{n}\right) /\left[z(z-1)\left(z-x_{1}\right)\left(z-x_{2}\right)\right], \quad z \in U,
$$

and $\xi=d \Phi(\mathbf{0})(\bar{\varphi} /|\varphi|)$ the corresponding projector $P_{\xi}^{*}$ possesses the following important property: $\varphi_{n} \in \operatorname{Ker} P_{\xi}^{*}$. Further, the norm

$$
f_{a}(t)=\iint_{U}\left|\varphi_{1}(z)+a \psi(z)+t \varphi_{n}(z)\right| d x d y, \quad t \in \boldsymbol{R},
$$


is considered for fixed real $a$. From the relation

$$
f_{a}(0)=\left\|P_{\varphi_{1}+a \psi}^{*}\left(\varphi_{1}+a \psi+t \varphi_{n}\right)\right\|_{A(U)} \leqq f_{a}(t)
$$

it follows on the basis of simple properties of integral (4) that for all $a \in \boldsymbol{R}$ the equality

$$
\operatorname{Re} \iint_{U} \varphi_{n} \frac{\bar{\varphi}_{1}+a \bar{\psi}}{\left|\varphi_{1}+a \psi\right|} d x d y=0
$$

must hold. However, direct calculation shows that for small $|a|$ the integral in the left-hand side of (5) is a fortiori distinct from zero.

4. Applications to univalent analytic functions. A) Let $D$ be a Jordan domain in $\overline{\boldsymbol{C}}, Q(D)$ be a class of normed univalent functions analytic in $D$, and let $Q_{k}(D)$ be its subclass of functions $k$-quasiconformally extendable on $\overline{\boldsymbol{C}}(0 \leqq k<1)$. Many necessary conditions for quasiconformal extendability are presented by inequalities for complex analytic functions $F_{\alpha}(f): Q(D) \rightarrow B$, where $B$ is a Banach space and $\alpha$ is a parameter belonging to an index set; of special interest is the case when a family $\left\{F_{\alpha}\right\}$ determines every $f$ uniquely. Let $F_{\alpha}$ also be well defined and holomorphic as a function of $S_{f}$. We assume that $F_{\alpha}(M)=0$ for any normalized Möbius-transformation $M$ in $Q(D)$, and that $\left\|F_{\alpha}(f)\right\| \leqq 1$ in $Q(D)$, where $\|\cdot\|$ is the norm in $B$. Then for $f \in Q_{k}(D)$ we have by Schwarz's lemma that

$$
\left\|F_{\alpha}(f)\right\| \leqq k, \quad \alpha \in A .
$$

Let us state the general problem: is the realization of the inequality (6) for all $\alpha$ also sufficient for the $k$-quasiconformal extendability of functions $f \in Q(D)$, i.e., does it follow from it that $f \in Q_{k}(D)$ ? One of its important special cases is the analogous question for Grunsky coefficient inequali'ies in the class $\Sigma(k)$ posed by $\mathrm{O}$. Lehto in [5]; its negative solution was obtained by R. Kühnau [3]. Theorem 2 gives the general result for any $F_{\alpha}$ :

Theorem 3. Let $D$ be a simply connected domain in $\overline{\boldsymbol{C}}$ bounded by a quasicircle. Then no sufficient condition of form (6) for the k-quasiconformal extendability of functions from $Q(D)$ can coincide with the necessary one, i.e., there always exists a function $f \in Q(D)$ for which $\left|F_{\alpha}(f)\right| \leqq k$ for all $\alpha \in A$, but $f \notin Q_{k}(D)$.

B) Let, as usual, $\Sigma(k)$ be a class of functions $f(z)=z+a_{1} z^{-1}+\ldots$ univalent in $\Delta^{*}=\{z \in \bar{C}:|z|>1\}$ with $k$-quasiconformal extension, and let $A(f)=\left(\sqrt{m n} \alpha_{m n}(f)\right)$, $m, n=1,2, \ldots$, be the Grunsky operator (matrix) for $f$ (see, for instance, [4]) which we regard as the operator $l^{2} \rightarrow l^{2}$. The following theorem essentially strengthens the area principle of the univalent function theory. 
Theorem 4. There is the exact estimate $\varrho(0,\|A(f)\|) \leqq c_{T}\left(\mathbf{0}, \boldsymbol{S}_{f}\right)$. Hence for any $f \in \Sigma(k)$ and $x=\left(x_{n}\right) \in l^{2}$ the inequality

$$
\left|\sum_{m, n=1}^{\infty} \sqrt{m n} \alpha_{m n}(f) x_{m} x_{n}\right| \leqq\left(\text { th } c_{T}\left(\mathbf{0}, S_{f}\right)\right) \sum_{n=1}^{\infty}\left|x_{n}\right|^{2} \leqq k \sum_{n=1}^{\infty}\left|x_{n}\right|^{2}
$$

holds; besides there exist $f \in \Sigma(k)$ for which the right-hand side of (7) is a strict inequality.

Outline of the proof. One can establish that $A(f)$ holomorphically maps $T$ into the unit ball $B\left(l^{2} \rightarrow l^{2}\right)$ of the space of linear automorphisms of $l^{2}$; therefore, due to the properties of the Carathéodory metric,

$$
c_{T}\left(\mathbf{0}, \boldsymbol{S}_{f}\right) \geqq c_{B\left(l^{2} \rightarrow l^{2}\right)}(\mathbf{0}, A(f))=\varrho(0,\|A(f)\|) .
$$

The last conclusion follows from Theorem 2 .

Theorem 1 gives analogues of Theorems 3 and 4 for analytic functions compatible with finitely generated quasi-Fuchsian groups of the first kind.

\section{References}

[1] KRUŠKAL', S. L.: Invariant metrics in Teichmüller spaces. - Sibirsk. Mat. Ž. 22: 2, 1981, 209212 (Russian).

[2] Kruschkal, S. L., und R. KüHNAU: Quasikonforme Abbildungen - neue Methoden und Anwendungen. - Teubner-Texte zur Math. 54, B. G. Teubner Verlagsgesellschaft, Leipzig, 1983.

[3] KüHnAU, R.: Zu dem Grunskyschen Koeffizientenbendingungen. - Ann. Acad. Sci. Fenn. Ser. A I Math. 6, 1981, 125-130.

[4] Lebedev, N. A.: The area principle in the univalent function theory. - Nauka, Moscow, 1975 (Russian).

[5] Lehto, O.: Quasiconformal mappings and singular integrals. - Sympos. Math. 18, Academic Press, New York - London, 1976, 429-453.

[6] Royden, H. L.: Automorphisms and isometries of Teichmüller spaces. - Advances in the theory of Riemann surfaces. Ann. of Math. Studies 66, Princeton Univ. Press, Princeton, N. J., 1971, 369-383.

Siberian Division of the USSR Academy of Sciences

Institute of Mathematics

Novosibirsk

USSR

Received 11 November 1983 